RICYDE. Revista Internacional de Ciencias del Deporte doi: $10.5232 /$ ricyde

Rev. int. cienc. deporte

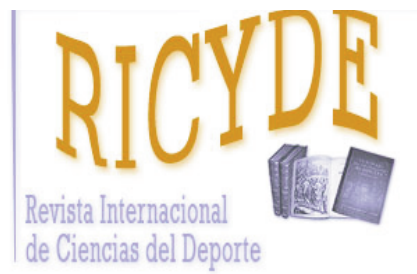

RICYDE. Revista Internacional de Ciencias del Deporte VOLUMEN XIV - AÑO XIV

Paginas:148-162 ISSN:1885-3137

Número: 52 - Abril - 2018

\title{
Orientaciones de meta como mediadoras en la relación entre perfeccionismo y ansiedad precompetitiva \\ Goal orientations as mediators in the relationship between perfectionism and precompetitive anxiety
}

\author{
Heriberto Antonio Pineda-Espejel, Edgar Alarcón, Zaida López-Ruiz y Marina Trejo \\ Universidad Autónoma de Baja California, Facultad de Deportes. Mexico
}

\section{Resumen}

El objetivo de este estudio fue analizar el efecto del perfeccionismo estado y las orientaciones de meta, sobre la ansiedad precompetitiva, a través de dos modelos que prueban las relaciones secuenciales. Un primer modelo analizó el efecto del perfeccionismo global en competición, sobre las orientaciones de meta, y consecuencias de ansiedad precompetitiva. Posteriormente, el perfeccionismo se separó en dos dimensiones, positiva y negativa, a partir de las facetas de esfuerzo por la perfección, y reacciones negativas ante la imperfección, respectivamente. Tal modelo probó la secuencia: dos facetas del perfeccionismo multidimensional en competición, orientaciones de meta, y consecuencias de ansiedad precompetitiva. Adicionalmente, en este segundo modelo se analizó el papel mediador de las orientaciones de meta. Participaron 171 deportistas de alto rendimiento, de ambos sexos, con una edad de 23.5 años $(D T=6.1)$, quienes respondieron a un conjunto de cuestionarios para medir las variables de estudio previo a una competición. Los modelos de ecuaciones estructurales mostraron que el perfeccionismo global predice la orientación al ego, y ésta la ansiedad cognitiva. No obstante, cuando dos dimensiones del perfeccionismo son diferenciadas, ambas facetas del perfeccionismo predicen positivamente la orientación al ego y los síntomas de ansiedad; contrariamente el esfuerzo por la perfección también predice positivamente la orientación a la tarea, y ésta negativamente ambas ansiedades. En conclusión, se refuerza que el perfeccionismo estado puede ser adaptativo, ya que el esfuerzo por la perfección en competición reduce los síntomas de ansiedad precompetitiva a través de la orientación a la tarea; por lo que las orientaciones de meta explican teóricamente la relación entre el esfuerzo por la perfección y la ansiedad precompetitiva.

Palabras clave: perfeccionismo estado; motivación; emoción; competición.

\section{Abstract}

The aim of this study was to analyze a predictive model of state perfectionism on goal orientations, and precompetitive anxiety and self-confidence, through two models that analyze sequential relationships. A first model analyzed the effect of global perfectionism in competition, on goal orientations, and consequences of precompetitive anxiety and self-confidence. Subsequently, perfectionism was separated into two dimensions, positive and negative, from the facets of striving for perfection, and negative reactions to imperfection, respectively. Such a model proved the sequence: two facets of multidimensional perfectionism in competition, goal orientations, and consequences of precompetitive anxiety and self-confidence. Additionally, the mediating role of goal orientations within the second model was analyzed. 171 high-performance athletes of both sexes, with an age of 23.5 years participated in the study, who answered a set of questionnaires to measure the study variables before the competition. The results of structural equation models showed that global perfectionism predicts ego orientation and cognitive anxiety. However, the two facets of perfectionism positively predict ego orientation and anxiety symptoms. Contrary to the striving for perfection also positively predicts the orientation in the task, and this negatively both anxieties. In conclusion, it is reinforced that state perfectionism can be an adaptive trait, since the striving for perfection, if accompanied by a predominance of task orientation. So the goal orientations theoretically explain the relationship between the striving for perfection and precompetitive anxiety.

Key words: state perfectionism; motivation; emotion; competition. 
Pineda-Espejel, H. A.; Alarcon, E.; López-Ruiz, Z., y Trejo, M. (2018). Orientaciones de meta como mediadoras en la relación entre perfeccionismo y ansiedad precompetitiva. RICYDE. Revista internacional de ciencias del deporte, 52(14), 148-162. https://doi.org/10.5232/ricyde2018.05205

\section{Introducción}

$\mathrm{A}$ lgunos autores consideran al perfeccionismo como un rasgo adaptativo que favorece el rendimiento en el deporte (Gould, Dieffenbach, y Moffett, 2002) y el aprendizaje de nuevas habilidades deportivas (Muñoz, González, y Olmedilla, 2016; Stoll, Lau, y Stoeber, 2008), por lo que actualmente existe controversia sobre el perfeccionismo, ya que en un inicio había sido considerado un rasgo de personalidad esencialmente negativo (Egan, Wade, y Shafran, 2011).

El perfeccionismo es una variable disposicional (Flett, Hewitt, Blankstein, y Dynin, 1994), que se define como una característica de la personalidad, determinada por el esfuerzo por la impecabilidad y el establecimiento de elevados estándares de rendimiento, acompañada por la tendencia a autoevaluaciones demasiado críticas por uno mismo y por los demás (Flett y Hewitt, 2002; Frost, Marten, Lahart, y Rosenblate, 1990).

Las investigaciones han sugerido que el perfeccionismo es multidimensional y multifacético, de forma que dos dimensiones pueden ser diferenciadas (Frost, Heimberg, Holt, Mattia, y Neubauer, 1993; Stoeber y Otto, 2006): una dimensión positiva o adaptativa llamada afanes perfeccionistas, representada por facetas de esfuerzo por la perfección, y altos estándares personales de rendimiento; y una dimensión negativa o poco adaptativa llamada preocupaciones perfeccionistas, que involucra facetas relacionadas con la preocupación por los errores (Hill y col., 2004), dudas sobre las acciones, sentimientos de discrepancia entre las expectativas y los resultados (Slaney, Rice, Mobley, Trippi, y Ashby, 2001), y reacciones negativas ante los errores.

El perfeccionismo positivo ha sido identificado como una característica psicológica común en deportistas que compiten en altos niveles (Gould y col., 2002), y se ha relacionado con consecuencias como afectos positivos (Stoeber, 2011), y orientación a la tarea (e.g. Appleton, Hall, y Hill, 2009). En contraste, la dimensión negativa del perfeccionismo se ha relacionado con consecuencias como la orientación al ego (e.g. Hall, Kerr, y Matthews, 1998) y la ansiedad (e.g. Frost y Henderson, 1991).

La evidencia antes mencionada, sugiere que el perfeccionismo se asocia con las orientaciones de meta. Apoyados en la teoría de las metas de logro (TML; Ames, 1992; Nicholls, 1989), las orientaciones de meta son las diferencias individuales en la forma de juzgar la competencia o habilidad. Desde la visión clásica de Nicholls (1989), se diferencian dos tipos: una orientación a la tarea, donde la habilidad se interpreta con base en el dominio de la ejecución o evidenciando maestría deportiva (Roberts, 2001); y una orientación al ego, donde para sentirse competente se necesita sobresalir de los demás o puntuar más.

En general, el perfeccionismo se asocia con la orientación al ego (Flett y Hewitt, 2002). No obstante, si se revisan por separado las dimensiones de afanes y preocupaciones perfeccionistas, en su relación con las orientaciones de meta, los afanes perfeccionistas se han relacionado positivamente con la orientación al ego (e.g. Lemyre, Hall, y Roberts, 2008), pero también lo han hecho con ambas orientaciones de meta (ego y tarea) (e.g. Appleton y col., 2009; Dunn, Causgrove Dunn, y Syrotuik, 2002; Hall y col., 1998; Ommundsen, Roberts, Lemyre, y Miller, 2005). Mientras que las preocupaciones perfeccionistas se han relacionado positivamente con la orientación al ego (e.g. Hall y col., 1998; Hall, Kerr, Kozub, y Finnie, 2007), negativamente con la orientación a la tarea (e.g. Dunn y col., 2002; Lemyre y col., 2008; Ommundsen y col., 2005), y en otros casos no han mostrado relación (e.g. Appleton y col., 2009). 
Pineda-Espejel, H. A.; Alarcon, E.; López-Ruiz, Z., y Trejo, M. (2018). Orientaciones de meta como mediadoras en la relación entre perfeccionismo y ansiedad precompetitiva. RICYDE. Revista internacional de ciencias del deporte, 52(14), 148-162. https://doi.org/10.5232/ricyde2018.05205

Por otra parte, la orientación al ego es a veces considerada como una orientación motivacional disfuncional, particularmente en combinación con baja orientación a la tarea (Duda, 2005), por lo que puede conducir a la ansiedad. La ansiedad estado competitiva es un estado emocional caracterizado por sentimientos de aprensión, tensión y activación en situaciones competitivas (Martens, Vealey, y Burton, 1990), la cual puede afectar el rendimiento deportivo (Burton, 1988; Smith, Smoll, y Schutz, 1990).

Martens y col. (1990) diferenciaron tres dimensiones en la experiencia competitiva: ansiedad cognitiva, que alude al componente mental causado por expectativas negativas o escasa confianza en las propias capacidades; ansiedad somática, que refiere a respuestas fisiológicas como tensión muscular; y autoconfianza, que alude al grado de seguridad sobre la capacidad de ser exitosos en su deporte (Weinberg y Gould, 2010). Si bien esta última no es una medida directa de ansiedad, alta autoconfianza antes de la competición es usualmente asociada con baja ansiedad competitiva (Craft, Magyar, Becker, y Fletz, 2003).

Diferentes características disposicionales, como las orientaciones de meta y el perfeccionismo anticipan la ansiedad estado competitiva (Cox, 2009). Por un lado, algunos estudios han relacionado las orientaciones de meta con la ansiedad precompetitiva, como Hall y col. (1998) quienes, en corredores de escuela secundaria, evidenciaron que la orientación a la tarea se relaciona negativamente con la ansiedad somática, y predice positivamente la autoconfianza, mientras que la orientación al ego se relaciona positivamente con la ansiedad cognitiva. Por su parte López y Pineda (2015) con deportistas universitarios probaron que la orientación al ego predice la ansiedad somática y cognitiva, y que la orientación a la tarea predice positivamente la autoconfianza y negativamente ambas ansiedades. Ruiz-Juan y Zarauz (2013) con atletas veteranos mostraron que, para su muestra total, la orientación al ego predijo positivamente la ansiedad cognitiva.

Por otro lado, respecto al perfeccionismo anticipando la ansiedad, Gucciardi, Mahoney, Jalleh, Donovan, y Parkes (2012) apuntaron que deportistas con predominio de perfeccionismo negativo tienen más predisposición hacia la ansiedad durante la competición. En el estudio de ansiedad competitiva y perfeccionismo, cuatro trabajos han medido las dos dimensiones del perfeccionismo (afanes y preocupaciones perfeccionistas). Frost y Henderson (1991), con deportistas universitarias, mostraron que el perfeccionismo global se relacionó positivamente con la ansiedad precompetitiva y negativamente con la autoconfianza. Hall y col. (1998), con deportistas estudiantiles, reflejaron que sólo la preocupación por los errores (faceta de la preocupación perfeccionista) se relacionó positivamente con la ansiedad precompetitiva e inversamente con la autoconfianza; mientras que los estándares personales (faceta de afanes perfeccionistas) se relacionaron positivamente con la autoconfianza.

Stoeber, Otto, Pescheck, Becker, y Stoll (2007) con deportistas universitarios, deportistas escolares y jugadoras de futbol de bajo nivel de rendimiento, obtuvieron que el perfeccionismo global se asoció con alta ansiedad cognitiva y somática precompetitivas. Sin embargo, las reacciones negativas ante la imperfección (faceta de la dimensión negativa) se asociaron positivamente con la ansiedad y negativamente con la autoconfianza, sin dejar clara la relación entre el esfuerzo por la perfección (faceta de la dimensión positiva) y la ansiedad y autoconfianza. Hamidi y Besharat (2010), con diferentes deportistas profesionales, demostraron que el esfuerzo por la perfección predijo negativamente las ansiedades cognitiva y somática competitivas, y se relacionó positivamente con la autoconfianza. Mientras que las reacciones negativas ante la imperfección predijeron positivamente la ansiedad somática, y se 
Pineda-Espejel, H. A.; Alarcon, E.; López-Ruiz, Z., y Trejo, M. (2018). Orientaciones de meta como mediadoras en la relación entre perfeccionismo y ansiedad precompetitiva. RICYDE. Revista internacional de ciencias del deporte, 52(14), 148-162. https://doi.org/10.5232/ricyde2018.05205

relacionaron positivamente con la ansiedad cognitiva, y en sentido inverso con la autoconfianza.

De los antecedentes, es notable que las asociaciones entre perfeccionismo, orientaciones de meta, y ansiedad y autoconfianza precompetitivas se han dado de forma parcelada, por lo que es necesario analizar las relaciones secuenciales entre dichas variables. En suma, Stoeber (2011) apuntó que se necesita saber más acerca de los mediadores potenciales en la relación entre el esfuerzo y preocupación perfeccionista y otras variables en el deporte.

Además, el perfeccionismo no solo es estable y persistente bajo algunas condiciones (i.e. rasgo de personalidad), si no que depende de una situación específica (i.e. estado) (Breding y Anshel, 2015; Saboonchi y Lundh, 1999), por lo que se puede decir que es más un estado que un rasgo (Breding y Anshel, 2015). Así que con deportistas sería necesario medir el perfeccionismo en competición, ya que ellos pueden focalizarse durante los entrenamientos en metas como entrenar efectivamente o divertirse, es decir una implicación a la tarea; mientras que durante la competición pueden focalizarse en metas como ganar o derrotar al oponente (Munroe-Chandler, Hall, y Weinberg, 2004), es decir una implicación al ego.

Por lo anterior, el objetivo de este estudio fue analizar un modelo predictivo del perfeccionismo estado sobre las orientaciones de meta, y ansiedad y autoconfianza precompetitivas, a través de dos modelos que analizan las relaciones secuenciales. El primer modelo analiza el papel predictor del perfeccionismo global en competición sobre las orientaciones de meta, y consecuencias de ansiedad y autoconfianza precompetitivas (Figura 1). El segundo modelo analiza el papel predictor de dos facetas del perfeccionismo multidimensional en competición (esfuerzo por la perfección, y reacciones negativas ante la imperfección) sobre las orientaciones de meta, y consecuencias de ansiedad y autoconfianza precompetitivas (Figura 2). Además, como objetivo específico se tiene probar el papel mediador de las orientaciones de meta en la relación entre las dos facetas del perfeccionismo en competición y consecuencias de ansiedad precompetitivas.

De acuerdo con los estudios previos, se hipotetiza que dentro del primer modelo, el perfeccionismo global predecirá positivamente la orientación al ego, y esta predecirá ambas ansiedades (cognitiva y somática) antes de la competición; además la orientación a la tarea predecirá positivamente la autoconfianza y negativamente ambas ansiedades (Figura 1). En el segundo modelo, se hipotetiza que el esfuerzo por la perfección predecirá positivamente ambas orientaciones de meta, y que las reacciones negativas ante la imperfección predecirán positivamente la orientación al ego y negativamente la orientación a la tarea; a su vez la orientación a la tarea predecirá positivamente la autoconfianza y negativamente ambas ansiedades, mientras que la orientación al ego predecirá positivamente ambas ansiedades antes de la competición (Figura 2).

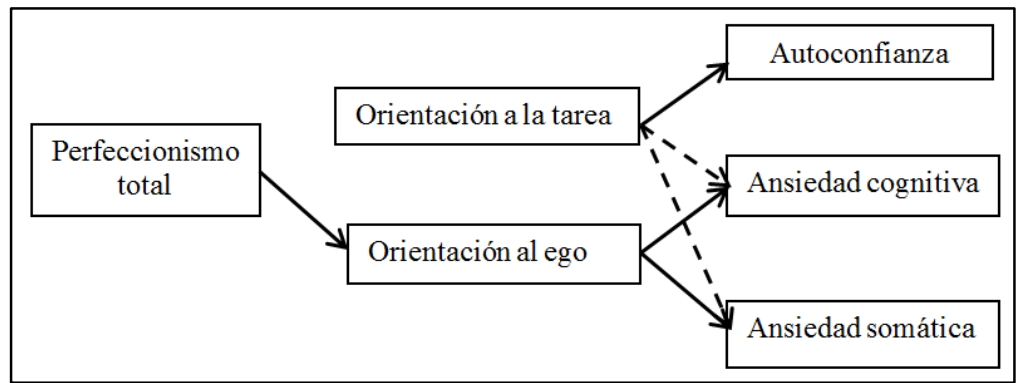

Figura 1. Modelo estructural hipotetizado para el perfeccionismo global en competición, orientaciones de meta, y ansiedad y autoconfianza precompetitivas. Las flechas continuas significan relación positiva, y las discontinuas representan una relación negativa. 
Pineda-Espejel, H. A.; Alarcon, E.; López-Ruiz, Z., y Trejo, M. (2018). Orientaciones de meta como mediadoras en la relación entre perfeccionismo y ansiedad precompetitiva. RICYDE. Revista internacional de ciencias del deporte, 52(14), 148-162. https://doi.org/10.5232/ricyde2018.05205

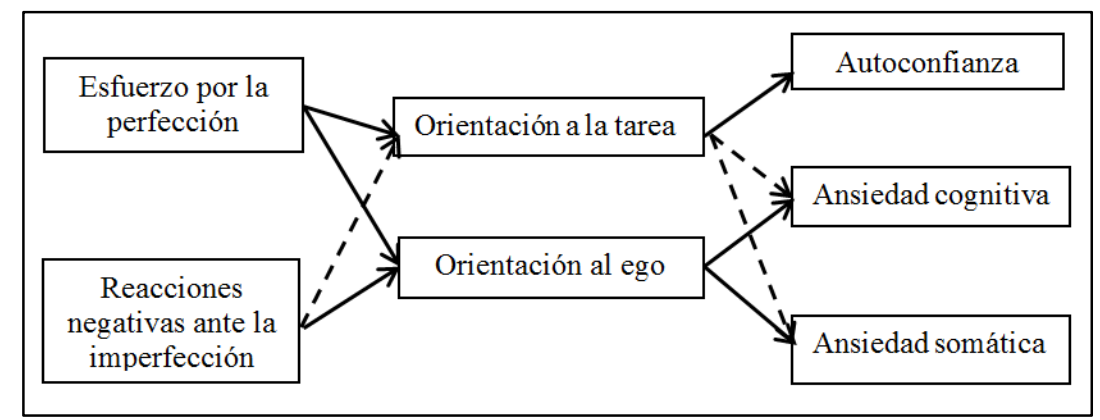

Figura 2. Modelo estructural hipotetizado para las facetas de esfuerzo por la perfección y reacciones negativas ante la imperfección en competición, orientaciones de meta, y ansiedad y autoconfianza precompetitivas. Las flechas continuas significan relación positiva, y las discontinuas representan una relación negativa.

\section{Método}

\section{Participantes}

Participaron 171 deportistas de alto rendimiento (76 hombres y 90 mujeres; cinco participantes no reportaron su sexo), con una edad promedio de 23.5 años $(D T=6.1)$, quienes compitieron en los XXII Juegos Centroamericanos y del Caribe 2014 en deportes individuales (e.g. esgrima, natación) y de conjunto (e.g. béisbol, futbol soccer), y representaban a diferentes países hispanohablantes (e.g. Colombia, Cuba, El Salvador, Guatemala, México, Puerto Rico, República Dominicana, Venezuela). Reportaron una antigüedad de entrenamiento promedio de 11.8 años $(D T=4.7)$, y entrenaban 4.4 horas diarias en promedio $(D T=2.4)$.

\section{Instrumentos}

Se utilizó la versión corta del Inventario Multidimensional de Perfeccionismo en el Deporte en competición adaptado al español (Pineda-Espejel, Alarcón, López-Walle, y Tomás-Marco, 2017) para medir el perfeccionismo en competición. Los ítems responden a la frase inicial "Durante la competición...", cinco de ellos capturan el esfuerzo por la perfección (e.g. "Tengo el deseo de hacer todo a la perfección"), y los cinco ítems restantes capturan las reacciones negativas ante la imperfección (e.g. "Me siento completamente furioso si cometo errores"). Se responden con una escala tipo Likert que va de nunca (1) a siempre (6). El índice global de perfeccionismo durante la competición se calculó combinando las puntuaciones de los ítems de esfuerzo por la perfección y de las reacciones negativas ante la imperfección.

Para medir las orientaciones de meta se utilizó el Cuestionario de Orientación al Ego y a la Tarea en el Deporte en su versión adaptada al contexto mexicano (López-Walle, Balaguer, Meliá, Castillo y Tristán, 2011). El cuestionario está compuesto por 13 ítems que responden a la frase inicial "Yo me siento con más éxito en mi deporte cuando...", y se divide en dos subescalas que miden la orientación a la tarea (e.g. "Aprendo un nuevo ejercicio y me impulsa a practicar más") y la orientación al ego (e.g. "Soy el único que puede hacer los ejercicios o técnicas en cuestión"). Las respuestas se hallan en una escala tipo Likert de cinco puntos que oscila desde muy en desacuerdo (1) a muy de acuerdo (5).

El Inventario de Ansiedad Estado Competitiva-2 Revisado, en su versión adaptada al contexto mexicano (Pineda-Espejel, López-Walle, y Tomás, 2014), fue utilizado para medir la intensidad de los síntomas de ansiedad precompetitiva y las percepciones de autoconfianza. Siete ítems miden la ansiedad somática (e.g. "Estoy muy inquieto"), cinco ítems miden la ansiedad cognitiva (e.g. "Me preocupa perder"), y el resto indagan sobre autoconfianza (e.g. 
Pineda-Espejel, H. A.; Alarcon, E.; López-Ruiz, Z., y Trejo, M. (2018). Orientaciones de meta como mediadoras en la relación entre perfeccionismo y ansiedad precompetitiva. RICYDE. Revista internacional de ciencias del deporte, 52(14), 148-162. https://doi.org/10.5232/ricyde2018.05205

“Tengo confianza de hacerlo bien”). Los ítems responden a la pregunta inicial “¿Cómo te sientes justo ahora antes de la competición?", y las respuestas se recogen en una escala tipo Likert de cuatro puntos que va desde nada (1) a mucho (4).

\section{Procedimiento}

La presente investigación se realizó de acuerdo con las directrices éticas propuestas por la American Psychological Association (APA). Vía telemática se solicitó la autorización a la Dirección General y a la Subdirección Técnico Deportiva de los XXII Juegos Centroamericanos y del Caribe 2014 para llevar a cabo el estudio. Se contó con el apoyo de la Subdirección de Servicios Médicos y Control de Dopaje para el acceso al recinto de competición.

El primer contacto personal fue con los entrenadores para solicitar el consentimiento de participación de sus deportistas. El protocolo de actuación para la obtención de datos fue similar en todos los participantes implicados en la investigación. La cumplimentación de los cuestionarios se realizó una hora antes de la competición en los recintos deportivos, en presencia de dos encuestadores para solventar las dudas en la comprensión de algún ítem. Igualmente se les explicó que responder a los cuestionarios, suponía aceptar la participación voluntaria en la investigación, y se les informó del anonimato y confidencialidad de los datos recogidos. En el caso de los deportistas menores de edad, el consentimiento de participación fue solicitado al entrenador o delegado del equipo.

\section{Análisis}

Análisis preliminares de normalidad, descriptivos y de fiabilidad (alfa de Cronbach) se efectuaron con el programa SPSS 22.0. Para poner a prueba los modelos hipotetizados se usaron modelos de ecuaciones estructurales con el programa LISREL 8.80 (Jöreskog y Sörbom, 2006). Dado el reducido tamaño muestral, el análisis se realizó con variables observadas, utilizando como método de estimación el de máxima verosimilitud robusta, y como input las matrices de correlaciones de Pearson y de covarianzas asintóticas (esta última para corregir la falta de normalidad de las variables). Para evaluar el ajuste entre los modelos teóricos planteados y la matriz de datos recogida se utilizó el Satorra-Bentler $\chi^{2}\left(\mathrm{~S}-\mathrm{B} \chi^{2}\right)$, así como los índices de bondad de ajuste incrementales NNFI, CFI, y RMSEA con un $90 \%$ de intervalo de confianza (IC 90\%). Para los índices incrementales NNFI y CFI valores iguales o mayores a .95 indican excelente ajuste (Hu y Bentler, 1999). El criterio para interpretar el índice RMSEA sugiere que valores menores o iguales a 0.05 indican un ajuste muy próximo al modelo (Browne y Cudeck, 1993).

Finalmente, se pusieron a prueba los efectos de mediación dentro del Modelo 2. Para evaluarlos se utilizó el método del producto de coeficientes (MacKinnon, Lockwood, Hoffman, West, y Sheets, 2002), en el que se ponía a prueba un modelo en el que, además de todos los efectos especificados en el modelo hipotetizado (Modelo 2), se incluía el efecto directo de la variable independiente sobre la variable dependiente $(\tau)$. Si los efectos de la variable independiente sobre la variable mediadora $(\alpha)$, y de la variable mediadora sobre la variable dependiente $(\beta)$ son significativos, se confirma el efecto de mediación. Para determinar si el producto $\alpha \beta$ es estadísticamente significativo se utiliza el valor crítico de la distribución del producto de variables aleatorias $(P>2.18 ; p<.05)$. En los casos en los que se confirmó el efecto de mediación, se evaluó si dicha mediación era total o parcial. Si el efecto directo $(\tau)$ resultaba ser estadísticamente significativo, se confirmaba la mediación parcial. En caso de que el efecto directo no resultara estadísticamente significativo, se confirmaba la mediación total. 
Pineda-Espejel, H. A.; Alarcon, E.; López-Ruiz, Z., y Trejo, M. (2018). Orientaciones de meta como mediadoras en la relación entre perfeccionismo y ansiedad precompetitiva. RICYDE. Revista internacional de ciencias del deporte, 52(14), 148-162. https://doi.org/10.5232/ricyde2018.05205

\section{Resultados}

Los resultados descriptivos (Tabla 1) ofrecieron que esta muestra reportó mayor orientación a la tarea, alto perfeccionismo global, a la par de alto esfuerzo por la perfección, así como alta autoconfianza. El esfuerzo por la perfección se relacionó positivamente con ambas orientaciones de meta; las reacciones negativas ante la imperfección lo hicieron con la orientación al ego y con ambas ansiedades. Finalmente la orientación al ego se relacionó positivamente con la ansiedad cognitiva, y la orientación a la tarea lo hizo con la autoconfianza.

Tabla 1. Estadísticos descriptivos, normalidad, consistencia interna, y matriz de correlación de Spearman de las variables

\begin{tabular}{|c|c|c|c|c|c|c|c|c|c|c|c|c|}
\hline & Rango & $M$ & $D T$ & $Z$ & 1 & 2 & 3 & 4 & 5 & 6 & 7 & 8 \\
\hline $\begin{array}{l}1 \text { Orientación a la } \\
\text { tarea }\end{array}$ & $1-5$ & 4.43 & 0.68 & $.16^{* *}$ & $(.74)$ & & & & & & & \\
\hline $\begin{array}{l}2 \text { Orientación al } \\
\text { ego }\end{array}$ & $1-5$ & 3.39 & 0.82 & $.07 * *$ & $.16^{*}$ & $(.79)$ & & & & & & \\
\hline $\begin{array}{l}3 \text { Ansiedad } \\
\text { cognitiva }\end{array}$ & $1-4$ & 1.95 & 0.73 & $.09 * *$ & -.08 & $.23 * *$ & $(.81)$ & & & & & \\
\hline $\begin{array}{l}4 \text { Ansiedad } \\
\text { somática }\end{array}$ & $1-4$ & 2.15 & 0.74 & $.09 * *$ & $-.21 * *$ & .09 & $.54 * *$ & $(.82)$ & & & & \\
\hline 5 Autoconfianza & $1-4$ & 3.15 & 0.63 & $.11 * *$ & $.35 * *$ & .00 & $-.19 * *$ & $-.23 * *$ & $(.80)$ & & & \\
\hline $\begin{array}{l}6 \text { Esfuerzo por la } \\
\text { perfección }\end{array}$ & $1-6$ & 4.86 & 1.06 & $.14 * *$ & $.20 * *$ & $.31 * *$ & .05 & .05 & $.23 * *$ & $(.89)$ & & \\
\hline $\begin{array}{l}7 \text { Reacciones } \\
\text { negativas ante la } \\
\text { imperfección }\end{array}$ & $1-6$ & 3.77 & 1.17 & $.10 * *$ & .00 & $.34 * *$ & $.29 * *$ & $.19 * *$ & -.00 & $.26 * *$ & $(.86)$ & \\
\hline $\begin{array}{l}8 \text { Índice global de } \\
\text { perfeccionismo }\end{array}$ & $1-6$ & 4.31 & 0.90 & $.06 * *$ & .14 & $.41 * *$ & $.23 * *$ & .13 & .13 & $.74 * *$ & $.80 * *$ & $(.85)$ \\
\hline
\end{tabular}

Nota: ${ }^{*} p<.01 ; * p<.05 ; M$ (promedio); DT (desviación típica); $Z$ (prueba de normalidad KolmogorovSmirnov); entre paréntesis fiabilidad con alfa de Cronbach.

\section{Modelos de ecuaciones estructurales}

El Modelo 1 evaluó la secuencia: perfeccionismo global en competición, orientaciones de meta, y ansiedad y autoconfianza precompetitivas. Las relaciones concretas definidas entre las variables del modelo aparecen en la Figura 1. Los resultados mostraron que el modelo tiene un alto poder explicativo, reflejando un ajuste excelente entre los datos empíricos y el modelo propuesto: $\mathrm{S}-\mathrm{B} \chi^{2}(6)=3.91 ; p>.05$; RMSEA $=.00(\mathrm{IC} 90 \%=.00-.07)$; NNFI $=$ 1.03; CFI $=1.0$. Los parámetros de la solución estandarizada (Figura 3) confirmaron parcialmente las relaciones planteadas dentro del modelo hipotetizado. El modelo propuesto representó el $19.9 \%$ de la varianza de la orientación al ego, el $8.7 \%$ de la varianza de ansiedad cognitiva, y el 7.5\% de la varianza de ansiedad somática. 
Pineda-Espejel, H. A.; Alarcon, E.; López-Ruiz, Z., y Trejo, M. (2018). Orientaciones de meta como mediadoras en la relación entre perfeccionismo y ansiedad precompetitiva. RICYDE. Revista internacional de ciencias del deporte, 52(14), 148-162. https://doi.org/10.5232/ricyde2018.05205

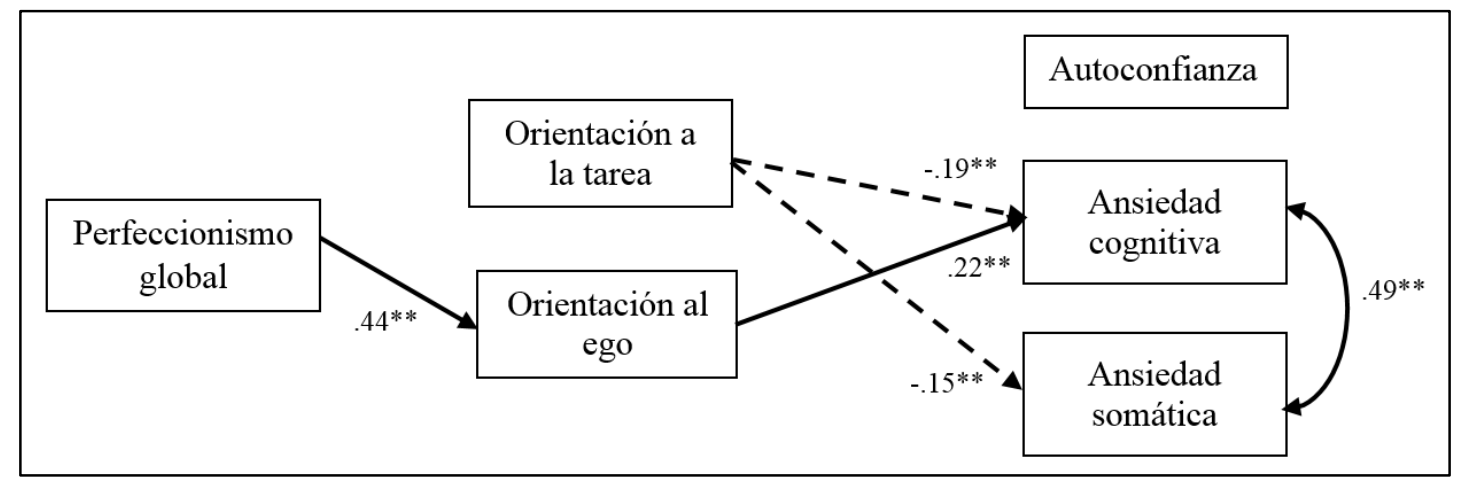

Nota: Todos los coeficientes están estandarizados y son significativos $\left({ }^{* *} p<.01\right)$. Se han excluido las predicciones no significativas.

Figura 3. Solución estandarizada del Modelo 1: perfeccionismo global en competición, orientaciones de meta, y ansiedad y autoconfianza precompetitivas.

El Modelo 2 evaluó la secuencia: esfuerzo por la perfección y reacciones negativas ante la imperfección en competición, orientaciones de meta, y ansiedad y autoconfianza precompetitivas. Las relaciones concretas definidas entre las variables del modelo aparecen en la Figura 2. Los resultados también mostraron un alto poder explicativo, y el modelo presentó un ajuste excelente: $\mathrm{S}-\mathrm{B} \chi^{2}(9)=7.11 ; p>.05$; RMSEA $=.00(\mathrm{IC} 90 \%=.00-.07)$; $\mathrm{NNFI}=1.02 ; \mathrm{CFI}=1.0$. Los parámetros de la solución estandarizada (Figura 4) confirmaron parcialmente las relaciones planteadas dentro del modelo hipotetizado. El modelo propuesto representó el $19.9 \%$ de la varianza de la orientación al ego, el $3.2 \%$ de la varianza de la orientación a la tarea, el $8.7 \%$ de la varianza de la ansiedad cognitiva, y el $7.5 \%$ de la ansiedad somática.

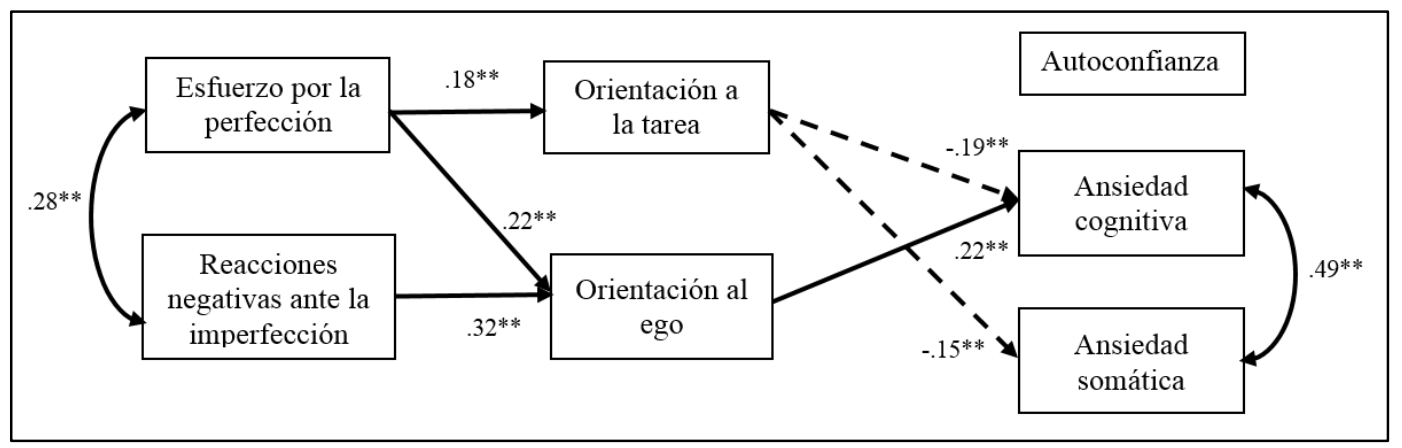

Nota: Todos los coeficientes están estandarizados y son significativos $(* * p<.01)$. Se han excluido las predicciones no significativas.

Figura 4. Solución estandarizada del Modelo 2: esfuerzo por la perfección y reacciones negativas ante la imperfección en competición, orientaciones de meta, y ansiedad y autoconfianza precompetitivas.

\section{Efectos de mediación}

Los efectos de mediación de las orientaciones de meta fueron calculados dentro del Modelo 2. Teniendo en cuenta los resultados presentados en el apartado anterior, se pusieron a prueba seis efectos en concreto. Dos efectos de mediación de la orientación a la tarea, 1) su mediación en la relación entre el esfuerzo por la perfección y la ansiedad cognitiva, el cual fue confirmado $(P=-15.9 ; p<.05)$ siendo un efecto total $(\tau=-.05 ; p>.05) ; 2)$ mediación en la relación entre el esfuerzo por la perfección y la ansiedad somática, mismo que fue confirmado $(P=-14.4 . p<.05)$ siendo una mediación total $(\tau=-.03 ; p>.05)$. 
Pineda-Espejel, H. A.; Alarcon, E.; López-Ruiz, Z., y Trejo, M. (2018). Orientaciones de meta como mediadoras en la relación entre perfeccionismo y ansiedad precompetitiva. RICYDE. Revista internacional de ciencias del deporte, 52(14), 148-162. https://doi.org/10.5232/ricyde2018.05205

Por otra parte, se evaluaron tres efectos de mediación de la orientación al ego. 1) Su efecto mediador en la relación entre el esfuerzo por la perfección y la ansiedad cognitiva, mismo que fue confirmado $(P=8.93 ; p<.05)$ siendo una mediación total $(\tau=.05 ; p>.05) ; 2)$ efecto en la relación entre el esfuerzo por la perfección y la ansiedad somática, el cual no se confirmó dado que el efecto $\beta$ no fue significativo. 3) su efecto en la relación entre las reacciones negativas ante la imperfección y la ansiedad cognitiva, el cual se confirmó $(P=$ $11.55 ; p<.05)$ siendo un efecto parcial $(\tau=.27 ; p<.01)$.

\section{Discusión}

Con el objetivo de diferenciar los patrones de las relaciones secuenciales que se dan a partir del perfeccionismo en el deporte, se pusieron a prueba dos modelos. Uno que analiza el papel predictor del perfeccionismo global en competición, sobre las orientaciones de meta y consecuencias de ansiedad y autoconfianza precompetitivas; y otro modelo que analiza el papel predictor de dos facetas del perfeccionismo multidimensional en competición (esfuerzo por la perfección, y reacciones negativas ante la imperfección), sobre las orientaciones de meta y consecuencias de ansiedad y autoconfianza precompetitivas.

Los resultados sugieren que el perfeccionismo en competición es una característica negativa, ya que se asocia con la orientación al ego, indicando que deportistas perfeccionistas se preocupan por metas de rendimiento, y descuidan metas de maestría deportiva, esto a partir del perfeccionismo global. Sin embargo, cuando dos dimensiones del perfeccionismo (afanes y preocupaciones perfeccionistas) son diferenciadas a través de las facetas de esfuerzo por la perfección, y de reacciones negativas ante la imperfección, la faceta de reacciones negativas ante la imperfección predice positivamente la orientación al ego, concordando con otros estudios (e.g. Hall y col., 1998, 2007); mientras que el esfuerzo por la perfección predice ambas orientaciones de meta (tarea y ego), coincidiendo con estudios previos que utilizaron muestras de deportistas con diferente nivel de rendimiento (e.g. Appleton y col., 2009; Dunn y col., 2002; Hall y col., 1998; Ommundsen y col., 2005).

Lo anterior sugiere que un deportista de alto rendimiento anticipándose a una competición puede pensar en esforzarse en todos los aspectos para un rendimiento perfectamente correcto, lo cual favorece a que se perciba competente o exitoso cuando domina la ejecución de los gestos motores técnicos o evidencia maestría deportiva, y que vea los errores como una fuente que proporciona información importante sobre cómo mejorar, de modo que su meta es esforzarse en la mejora personal durante la competición. Pero el mismo esfuerzo por desempeñarse perfectamente en competición, también favorece que para percibirse competente necesite mostrar superioridad frente a los demás, por lo que su meta es ganar o puntuar más que otros. De manera que el esfuerzo por la perfección es un motor para lograr la meta en competición, ya sea dar el mayor esfuerzo, o ganar.

Por otra parte, un deportista de alto rendimiento que se anticipa a la misma competición puede frustrarse o molestarse consigo mismo cuando no logra ejecuciones perfectas, lo que conduce a que se perciba competente sólo cuando demuestra que es superior a los demás, o iguales pero realizando menor esfuerzo. Esto se debe a que las reacciones negativas ante la imperfección y la orientación al ego están estrechamente ligadas a evaluar el propio desempeño deportivo mediante criterios normativos como ganar.

En este sentido, se refuerza que las tendencias perfeccionistas pueden ser consideradas parte de un modo de motivación y de esfuerzo para el logro (Duda y Hall, 2001) en competición, ya sea focalizándose en metas como ganar, derrotar al oponente (Munroe-Chandler y col., 2004), o esforzarse por realizar los gestos motores técnicos y tácticos con maestría deportiva. 
Pineda-Espejel, H. A.; Alarcon, E.; López-Ruiz, Z., y Trejo, M. (2018). Orientaciones de meta como mediadoras en la relación entre perfeccionismo y ansiedad precompetitiva. RICYDE. Revista internacional de ciencias del deporte, 52(14), 148-162. https://doi.org/10.5232/ricyde2018.05205

En suma, los deportistas que se esfuerzan por la impecabilidad de sus técnicas deportivas en competición, y se enfocan en mostrar maestría deportiva, experimentan menos síntomas de tensión muscular o sudoración en manos antes de la competición, así como menores pensamientos e imágenes negativas sobre su actuación en competición, lo que reduce la preocupación e inseguridad en su capacidad de rendimiento, porque quienes mayormente definen el éxito en su deporte en términos autoreferidos, son menos propensos a experimentar excesiva ansiedad (Roberts, 2001); guardando relación con aproximaciones de estudios previos (e.g. Hall y col., 1998).

Mientras que quienes se esfuerzan por la impecabilidad en competición, pero mayormente se enfocan en ganar o mostrar superioridad, manifiestan más pensamientos e imágenes negativas sobre el desempeño, conduciendo a experimentar preocupación, temor e inseguridad en su rendimiento, y por tanto dificultad en la atención (Burton, 1988). Ello confirmado a partir del análisis de mediación.

De forma similar, deportistas que tienden a frustrarse o enfadarse consigo mismos cuando no ejecutan los gestos técnico-tácticos con perfección en competición, tengan o no un predominio de orientación al ego, manifiestan elevada ansiedad cognitiva previo a una competición. Esto concuerda con aproximaciones de estudios previos (e.g. Gucciardi y col., 2012; Hall y col., 1998; López y Pineda, 2015), y puede obedecer a que las reacciones negativas ante la imperfección se relacionan con la preocupación por los errores (Frost y Henderson, 1991), por tanto, hacen que tengan expectativas negativas sobre el propio rendimiento deportivo. Aunque en el deporte de alto rendimiento, en ocasiones se espera que los deportistas ejecuten técnicas o jugadas perfectas, el reaccionar negativamente ante la imperfección puede deteriorar el rendimiento deportivo porque conduce a mayores pensamientos de tipo catastrófico.

Si bien, a nivel correlacional la autoconfianza se asocia positiva y significativamente con el esfuerzo por la perfección y con la orientación a la tarea, concordando con otros estudios (e.g. Hall y col., 1998; Hamidi y Besharat, 2010; López y Pineda, 2015), de forma secuencial se pierde el efecto de predicción, lo que puede deberse a los antecedentes del perfeccionismo en competición, ya que las asociaciones positivas del esfuerzo por la perfección, a veces se suprimen por las asociaciones negativas de las reacciones negativas ante la imperfección.

Los hallazgos apoyan la consideración extendida de que los deportistas de alto nivel presentan puntajes elevados en orientación a la tarea y al ego. Además denotan que en esta muestra de estudio el esfuerzo por la perfección se relaciona positivamente con las reacciones negativas ante la imperfección, es decir, que los deportistas que se esfuerzan por ejecutar las técnicas a la perfección durante la competición, también son susceptibles a frustrarse o molestarse consigo mismos cuando no logran actuaciones perfectas. Esto apoya que en el deporte los afanes perfeccionistas usualmente muestran relaciones positivas con las preocupaciones perfeccionistas (Stoeber y col., 2007, 2009). No obstante, la muestra reportó más esfuerzo por la perfección que reacciones negativas ante la imperfección, concordando con que el perfeccionismo adaptativo ha sido identificado como una característica psicológica común en deportistas que compiten en altos niveles (Gould y col., 2002).

Este trabajo tiene implicaciones teóricas. Si bien las relaciones entre las variables de estudio se han analizado previamente, esto ha sido de forma parcelada y a partir del perfeccionismo como rasgo; mientras que este trabajo las analizó de forma secuencial, midiendo el perfeccionismo como un estado, y añadiendo el papel mediador de las orientaciones de meta; con lo que aporta que ambas orientaciones de meta explican teóricamente la relación entre el esfuerzo por la perfección en competición y la ansiedad precompetitiva. Desde este enfoque, 
también se apoya que el perfeccionismo es multidimensional y multifacético, concordando con lo apuntado por Chang (2003) de que sólo la faceta de reacciones negativas ante la imperfección es claramente poco adaptativa, mientras que el esfuerzo por la perfección en el deporte puede tener connotaciones adaptativas. Así, las dos dimensiones del perfeccionismo son distinguibles conceptual y estadísticamente, demostrando que es importante diferenciar entre afanes y preocupaciones perfeccionistas cuando se investiga el perfeccionismo en el deporte, puesto que es una característica ambivalente.

También tiene implicaciones prácticas para los deportistas, entrenadores y psicólogos del deporte, porque los resultados, en línea con Flett y Hewitt (2005), muestran que el perfeccionismo no es precisamente una característica negativa que afecte las emociones o cogniciones previas a una competición. Los esfuerzos perfeccionistas pueden ayudar a tener menos pensamientos e imágenes de tipo catastrófico siempre y cuando predomine una orientación a la tarea. Así mismo, sugieren que en el deporte no debería incrementarse el esfuerzo por la perfección, sino controlar las reacciones negativas ante la imperfección de los deportistas, así como fomentar la definición de éxito en términos autoreferidos, siendo una tarea para los psicólogos del deporte.

Dentro de las limitaciones del estudio se tienen el reducido tamaño muestral, por lo que estos resultados no pueden generalizarse al alto rendimiento ni a otros niveles de rendimiento. Además, sólo se midieron dos facetas del perfeccionismo mutidimensional, por lo que es importante que futuros estudios empleen diferentes mediciones multidimensionales del perfeccionismo en el deporte e investiguen otras facetas positivas y negativas del perfeccionismo. Los resultados de este estudio pudieron verse influenciados por las diferentes nacionalidades y culturas de la muestra; no obstante, aunque existen variedades del español entre los países hispanoamericanos, la lengua española conserva su unidad básica, y los cambios gramaticales son menos notables, por lo que el día de hoy es básicamente una sola lengua la que se habla y escribe en las excolonias (Alatorre, 2008), además, los instrumentos utilizados demostraron adecuada consistencia interna en la muestra de estudio.

\section{Conclusión}

En conclusión el perfeccionismo es en sí mismo un constructo paradójico. El perfeccionismo estado puede ser adaptativo, puesto que el esforzarse por la impecabilidad de los gestos técnico-tácticos conduce a menor ansiedad somática y cognitiva antes de la competición, sólo si el deportista concibe los errores como una oportunidad de mejora, se percibe competente mediante el dominio de los ejercicios o jugadas, o se define exitoso cuando da su mayor esfuerzo. Contrariamente, el esforzarse por la impecabilidad en competición aumenta la ansiedad cognitiva sólo si el deportista se define competente mediante criterios normativos. En tanto que las reacciones negativas ante la imperfección representan un aspecto poco adaptativo del perfeccionismo, porque aumentan las percepciones de ansiedad cognitiva, se acompañe o no de una orientación al ego.

\section{Agradecimientos}

Este trabajo se ha realizado, en parte, gracias a la subvención 149/4/N/7/1 de la Primera Convocatoria Interna de Proyectos de Investigación Especial de la Universidad Autónoma de Baja California. 
Pineda-Espejel, H. A.; Alarcon, E.; López-Ruiz, Z., y Trejo, M. (2018). Orientaciones de meta como mediadoras en la relación entre perfeccionismo y ansiedad precompetitiva. RICYDE. Revista internacional de ciencias del deporte, 52(14), 148-162. https://doi.org/10.5232/ricyde2018.05205

\section{Referencias}

Ames, C. (1992). Classrooms: goals, structures and student motivation. Journal of Educational Psychology, 84(3), 261-271. Ames, C. (1992). Classrooms: goals, structures and student motivation. Journal of Educational Psychology, 84(3), 261271. https://doi.org/10.1037/0022-0663.84.3.261

Appleton, P. R.; Hall, H. K., \& Hill, A. P. (2009). Relations between multidimensional perfectionism and burnout in junior-elite male athletes. Psychology of Sport and Exercise, 10(4), 457-465.

https://doi.org/10.1016/j.psychsport.2008.12.006

Breeding, T. W., \& Anshel, M. H. (2015). Relationship between sport perfectionism and perceived competence as a function of skill level and sport type. Journal of Sport Behavior, 38(4), 376.

Browne, M. W., \& Cudeck, R. (1993). Alternative ways of assessing model fit. En K. A. Bollen y J. S. Long (Eds.), Testing Structural Equation Models (pp. 136-162). Newbury Park, CA.: SAGE.

Burton, D. (1988). Do anxious swimmers swim slower? Reexamining the elusive anxietyperformance relationships. Journal of Sports and Exercise Psychology, 10(1), 45-61. https://doi.org/10.1123/jsep.10.1.45

Chang, E. C. (2003). On the perfectibility of the individual: Going beyond the dialectic of good versus evil. En E. C. Chang y L. J. Sanna (Eds.), Virtue, vice, and personality: The complexity of behavior (pp. 125-144). Washington, DC: American Psychological Association. https://doi.org/10.1037/10614-008

Cox, R. H. (2009). Psicología del deporte: Conceptos y sus aplicaciones. Madrid: Médica Panamericana.

Craft, L. L.; Magyar, T. M.; Becker, B. J., \& Feltz, D. L. (2003). The relationship between the Competitive State Anxiety Inventory-2 and sport performance: A Meta-Analysis. Journal of Sport and Excercise Psychology, 25(1), 44-65. https://doi.org/10.1123/jsep.25.1.44

Duda, J. L. (2005). The relevance of competence and achievement goals. En A. J. Elliot y C. S. Dweck (Eds.), Handbook of competence and motivation (pp. 318-335). New York: Guiford.

Duda, J. L., \& Hall, H. (2001). Achievement goal theory in sport: Recent extensions and future directions. En R. N. Singer, H. A. Hausenblas y C. M. Janelle (Eds.), Handbook of sport psychology (pp. 417 - 443). New York: Wiley.

Dunn, J. G. H.; Causgrove Dunn, J., \& Syrotuik, D. G. (2002). Relationship between multidimensional perfectionism and goal orientations in sport. Journal of Sport and Exercise Psychology, 24(4), 376-395. https://doi.org/10.1123/jsep.24.4.376

Egan, S. J.; Wade, T. D., \& Shafran, R. (2011). Perfectionism as a transdiagnostic process: A clinical review. Clinical Psychology Review, 31(2), 203-212.

https://doi.org/10.1016/j.cpr.2010.04.009

Flett, G. L., \& Hewitt, P. L. (2002). Perfectionism and maladjustment: An overview of theoretical, definitional, and treatment issues. En G. L. Flett y P. L. Hewitt (Eds.), Perfectionism: Theory, research, and treatment, (pp. 5-31). Washington, DC: American Psychological Association. https://doi.org/10.1037/10458-001 
Pineda-Espejel, H. A.; Alarcon, E.; López-Ruiz, Z., y Trejo, M. (2018). Orientaciones de meta como mediadoras en la relación entre perfeccionismo y ansiedad precompetitiva. RICYDE. Revista internacional de ciencias del deporte, 52(14), 148-162. https://doi.org/10.5232/ricyde2018.05205

Flett, G. L., \& Hewitt, P. L. (2005). The perils of perfectionism in sports and exercise. Current Directions in Psychological Science, 14(1), 14-18. https://doi.org/10.1111/j.0963-7214.2005.00326.x

Flett, G. L.; Hewitt, P. L.; Blankstein, K. R., \& Dynin, C. B. (1994). Dimensions of perfectionism and Type A behaviour. Personality and Individual Differences, 16(3), 477-485. https://doi.org/10.1016/0191-8869(94)90073-6

Frost, R. O., \& Henderson, K. J. (1991). Perfectionism and reactions to athletic competition. Journal of Sport and Exercise Psychology, 13(4), 323-335. https://doi.org/10.1123/jsep.13.4.323

Frost, R. O.; Heimberg, R. G.; Holt, C. S.; Mattia, J. I., \& Neubauer, A. L. (1993). A comparison of two measures of perfectionism. Personality and Individual Differences, 14(1), 119-126. https://doi.org/10.1016/0191-8869(93)90181-2

Frost, R. O.; Marten, P.; Lahart, C., \& Rosenblate, R. (1990). The dimensions of perfectionism. Cognitive Therapy and Research, 14(5), 449-468. https://doi.org/10.1007/BF01172967

Gould, D.; Dieffenbach, K., \& Moffett, A. (2002). Psychological characteristics and their development in Olympic champions. Journal of Applied Sport Psychology, 14(3), 172204. https://doi.org/10.1080/10413200290103482

Gucciardi, D. F.; Mahoney, J.; Jalleh, J.; Donovan, R. J., \& Parkes, J. (2012). Perfectionistic profiles among elite athletes and differences in their motivational orientations. Journal of Sport and Exercise Psychology, 34(2), 149-183. https://doi.org/10.1123/jsep.34.2.159

Hall, H. K.; Kerr, A. W., \& Matthews, J. (1998). Precompetitive anxiety in sport: The contribution of achievement goals and perfectionism. Journal of Sport and Exercise Psychology, 20(2), 194-217. https://doi.org/10.1123/jsep.20.2.194

Hall, H. K.; Kerr, A. W., Kozub, S. A., \& Finnie, S. B. (2007). Motivational antecedents of obligatory exercise: The influence of achievement goals and multidimensional perfectionism. Psychology of Sport and Exercise, 8(3), 297-316. https://doi.org/10.1016/j.psychsport.2006.04.007

Hamidi, S., \& Besharat, M. A. (2010). Perfectionism and competitive anxiety in athletes. Procedia-Social and Behavioral Sciences, 5, 813-817. https://doi.org/10.1016/j.sbspro.2010.07.190

Hill, R. W.; Huelsman, T. J.; Furr, R. M.; Kibler, J.; Vicente, B. B., \& Kennedy, C. (2004). A new measure of perfectionism: The Perfectionism Inventory. Journal of Personality Assessment, 82(1), 80-91. https://doi.org/10.1207/s15327752jpa8201_13

Hu, L. T., \& Bentler, P. M. (1999). Cutoff criteria for fit indexes in covariance structure analysis: Conventional criteria versus new alternatives. Structural Equation Modeling: A Multidisciplinary Journal, 6(1), 1-55. https://doi.org/10.1080/10705519909540118

Jöreskog, K. G., \& Sörbom, D. (2006). Lisrel 8.80 [Computer software]. Chicago: Scientific Software International.

Lemyre, P.-N., Hall, H. K., \& Roberts, G. C. (2008). A social cognitive approach to burnout in athletes. Scandinavian Journal of Medicine and Science in Sports, 18(2), 221-234.

https://doi.org/10.1111/j.1600-0838.2007.00671.x 
López, J., y Pineda, A. (2015). Orientaciones de meta, intensidad y dirección de ansiedad precompetitiva en deportistas universitarios. Ciencia UANL, 18(74), 44-50.

López-Walle, J.; Balaguer, I., Meliá, J. L.; Castillo, I., y Tristán, J. (2011). Adaptación a la población mexicana del Cuestionario de Orientación al Ego y a la Tarea en el Deporte (TEOSQ). Revista de Psicología del Deporte, 20(2), 523-536.

MacKinnon, D. P.; Lockwood, C. M.; Hoffman, J. M.; West, S. G., \& Sheets, V. (2002). A comparison of methods to test mediation and other intervening variable effects. Psychological Methods, 7(1), 83-104. https://doi.org/10.1037/1082-989X.7.1.83

Martens, R.; Vealey, R. S., \& Burton, D. (1990). Competitive anxiety in sport. Champaign, IL: Human Kinetics Publishers.

Muñoz, A. J.; González, J., y Olmedilla, A. (2016). Perfeccionismo percibido y expresión de ira en deportistas varones adolescentes: Influencia sobre la autoestima. Revista Argentina de Ciencias del Comportamiento, 8(3), 30-39.

Munroe-Chandler, K. J.; Hall, C. R., \& Weinberg, R. S. (2004). A qualitative analysis of the types of goals athletes set in training and competition. Journal of Sport Behavior, 27(1), 58-74.

Nicholls, J. G. (1989). The competitive ethos and democratic education. Cambridge, ASS: Harvard University.

Ommundsen, Y.; Roberts, G. C.; Lemyre, P.-N., \& Miller, B. W. (2005). Peer relationships in adolescent competitive soccer: Associations to perceived motivational climate, achievement goals and perfectionism. Journal of Sports Sciences, 23(9), 977989. https://doi.org/10.1080/02640410500127975

Pineda-Espejel, A.; Alarcón, E. I.; López-Walle, J., y Tomás-Marco, I. (2017). Adaptación al español de la versión corta del Inventario de Perfeccionismo Multidimensional en el Deporte en competición. Revista Iberoamericana de Diagnóstico y Evaluación - e Avaliação Psicológica, 43(1), 45-57. https://doi.org/10.21865/RIDEP43_45

Pineda-Espejel, H. A.; López-Walle, J., y Tomás, I. (2014). Validación de la versión mexicana del CSAI-2R en sus escalas de intensidad y dirección. Revista Mexicana de Psicología, 31(2), 198-212.

Roberts, G. (2001). Advances in motivation in sport and exercise. Champaign IL: Human Kinetics.

Ruiz-Juan, F., y Zarauz, A. (2013). Ansiedad, satisfacción, percepción y creencias sobre las causas del éxito en atletas veteranos españoles. Revista de Ansiedad y Estrés, 19(1), 83-93.

Saboonchi, F., \& Lundh, L. G. (1999). State perfectionism and its relation to trait perfectionism, type of situation, priming, and being observed. Scandinavian Journal of Behaviour Therapy, 28(4), 154-166.

https://doi.org/10.1080/028457199439900

Slaney, R. B.; Rice, K. G., Mobley, M.; Trippi, J., \& Ashby, J. S. (2001). The Revised Almost Perfect Scale. Measurement and Evaluation in Counseling and Development, 34(3), 130-145.

Smith, R. E.; Smoll, F. L., \& Schutz, R. W. (1990). Measurement and correlates of sport specific cognitive and somatic trait anxiety: The Sport Anxiety Scale. Anxiety Research, 2(4), 263-280.

https://doi.org/10.1080/08917779008248733 
Stoeber, J. (2011). The dual nature of perfectionism in sports: Relationships with emotion, motivation, and performance. International Review of Sport and Exercise Psychology, 4(2), 128-145.

https://doi.org/10.1080/1750984X.2011.604789

Stoeber, J., \& Otto, K. (2006). Positive conceptions of perfectionism: Approaches, evidence, challenges. Personality and Social Psychology Review, 10(4), 295-319. https://doi.org/10.1207/s15327957pspr1004_2

Stoeber, J.; Otto, K.; Pescheck, E.; Becker, C., \& Stoll, O. (2007). Perfectionism and competitive anxiety in athletes: Differentiating striving for perfection and negative reactions to imperfection. Personality and Individual Differences, 42(6), 959-969. https://doi.org/10.1016/j.paid.2006.09.006

Stoll, O.; Lau, A., \& Stoeber, J. (2008). Perfectionism and performance in a new basketball training task: Does striving for perfection enhance or undermine performance? Psychology of sport and Exercise, 9(5), 620-629. https://doi.org/10.1016/j.psychsport.2007.10.001

Weinberg, R. S., \& Gould, D. (2010). Fundamentos de psicología del deporte y del ejercicio físico. Madrid: Médica Panamericana. 